\title{
Terpinen-4-ol inhibits colorectal cancer growth via reactive oxygen species
}

\author{
KEN NAKAYAMA ${ }^{1}$, SOICHIRO MURATA $^{2}$, HIROMU ITO $^{3}$, KENICHI IWASAKI $^{1}$, \\ MYRA ORLINA VILLAREAL ${ }^{4}$, YUN-WEN ZHENG ${ }^{1}$, HIROFUMI MATSUI $^{3}$, \\ HIROKO ISODA $^{4}$ and NOBUHIRO OHKOHCHI ${ }^{1}$
}

\begin{abstract}
${ }^{1}$ Department of Surgery, Division of Gastroenterological and Hepatobiliary Surgery, and Organ Transplantation, Faculty of Medicine, University of Tsukuba, Tsukuba, Ibaraki 305-8575; ${ }^{2}$ Department of Regenerative Medicine, Yokohama University, Yokohama, Kanagawa 236-0004; ${ }^{3}$ Department of Gastroenterology, Faculty of Medicine, University of Tsukuba, Tsukuba, Ibaraki 305-8575; ${ }^{4}$ Faculty of Life and Environmental Sciences, University of

Tsukuba Alliance of Research on North Africa, University of Tsukuba, Tsukuba, Ibaraki 305-8572, Japan
\end{abstract}

Received August 25, 2016; Accepted March 19, 2017

DOI: $10.3892 / 01.2017 .6370$

\begin{abstract}
Terpinen-4-ol (TP4O) is the main component of the essential oil extracted from Melaleuca alternifolia, known as the tea tree, of the botanical family Myrtaceae. The anticancer effects of TP4O have been reported in several cancer cell lines. Previous reports have demonstrated that TP4O exerts anticancer effects by inducing apoptotic cell death in several cell lines; however, the underlying molecular mechanisms of these effects remain unclear. In the present study, the anticancer effects of TP4O against the colorectal cancer (CRC) cell lines HCT116 and RKO were evaluated using WST-8 and bromodeoxyuridine assays. The mechanism of cell death was investigated by the measurement of caspase-3/7, Annexin V and lactate dehydrogenase release. Reactive oxygen species (ROS) levels induced by TP4O were evaluated by electron spin resonance and quantitative measurement of dihydroethidium. Localization of the ROS derived from mitochondria was observed by confocal inverted microscopy. Protein levels of ROS scavengers were assessed by western blotting analysis. To confirm the role of ROS, cell viability was measured in the presence of antioxidant reagents. In an in vivo xenograft model of ICR-SCID mice implanted with HCT116 cells, $200 \mathrm{mg} / \mathrm{kg}$ TP4O was injected locally, and tumor growth was compared with that of the control. TP4O induced apoptotic cell death in HCT116 and RKO cells in a dose-dependent manner, and TP4O also increased the levels of ROS generated
\end{abstract}

Correspondence to: Professor Nobuhiro Ohkohchi, Department of Surgery, Division of Gastroenterological and Hepatobiliary Surgery, and Organ Transplantation, Faculty of Medicine, University of Tsukuba, 1-1-1 Tennodai, Tsukuba, Ibaraki 305-8575, Japan E-mail: nokochi3@md.tsukuba.ac.jp

Key words: colorectal cancer, terpinen-4-ol, reactive oxygen species, apoptosis, monoterpene, electron spin resonance by mitochondria. TP4O-induced cell death was rescued by administration of antioxidant regents. In vivo, TP4O inhibited the proliferation of HCT116 xenografts compared with that of the control group. The results of the present study suggest that TP4O induces apoptosis in CRC cells through ROS generation. Furthermore, TP4O is potentially useful for the development of novel therapies against CRC.

\section{Introduction}

Colorectal cancer (CRC) is the third most common cancer in males and the second in females worldwide (1). For patients in the early stages of CRC, i.e., stages I and II, surgery is the most common treatment, and the 5-year relative survival for patients with early-stage CRC is $90.3 \%$. By contrast, for patients with advanced $\mathrm{CRC}$, chemotherapy and/or radiation therapy is required, and the 5-year survival rate of patients with distant metastasis is $12.5 \%$ (2). The ideal anticancer drug would be one that is affordable, has few side effects and is sufficiently potent to achieve complete remission. Inhibitors of vascular endothelial growth factor and monoclonal antibodies that inhibit epidermal growth factor receptor have prolonged the survival of advanced CRC patients $(3,4)$. However, these drugs have severe side effects, and thus, researchers have been searching for novel biochemical compounds (5). Natural products have become an important resource for novel drug discovery (5), and $>50 \%$ of anticancer drugs are derived from natural products (6).

Essential oils are natural products extracted from seeds, leaves and tree resin. These natural oils are used primarily in perfumes, cosmetics and the food industry (7). Various of these oils have been demonstrated to possess antibiotic, anti-inflammatory and anticancer properties $(7,8)$. The molecular mechanisms underlying their anticancer effects have been revealed to occur via reactive oxygen species (ROS) signaling pathways and cell cycle arrest $(9,10)$. Terpinen-4-ol (TP4O) is the main component of the essential oil extracted from Melaleuca alternifolia (of the botanical family Myrtaceae), a 
plant native to Australia that is also known as the tea tree (11). Tea tree oil (TTO) is famous for its scent, and has been demonstrated to have antibacterial $(11,12)$ and anti-inflammatory properties (13). Previous studies have investigated the anticancer effect of TP4O against human melanoma cells (14), human non-small cell lung cancer (15), human leukaemia cells (16) and CRC cells (17). However, the anticancer effects of TPO4 remain unclear. In the present study, the anticancer effects of TP40 against CRC cells, in particular the role of ROS, were evaluated using the HCT116 and RKO cell lines.

\section{Materials and methods}

Chemicals and reagents. TP4O, Triton X-100, staurosporine and ebselen (EB) were purchased from Sigma-Aldrich (Merck KGaA, Darmstadt, Germany). TP4O was dissolved in ethanol to prepare stock solutions. The compound was administered at a final concentration of $0.0165 \%$ ethanol. High-glucose (4.5 g/l) Dulbecco's modified Eagle's medium (DMEM), phenol red-free DMEM and PBS were purchased from Wako Pure Chemical Industries, Ltd. (Osaka, Japan). Eagle's minimum essential medium (EMEM) was purchased from the American Type Culture Collection (ATCC; Manassas, VA, USA). 5,5-dimethyl-1-pyrroline-N-oxide (DMPO) was purchased from Dojindo Molecular Technologies, Inc. (Kumamoto, Japan). N-acetyl-L-cysteine (NAC) and manganese (III) tetrakis (4-benzoic acid) porphyrin chloride (MnTBAP) were purchased from EMD Millipore (Billerica, MA USA) and Funakoshi Co., Ltd. (Tokyo, Japan), respectively. Phenylmethylsulfonyl fluoride was purchased from Roche Diagnostics GmbH (Mannheim, Germany).

Cell lines and cell culture. Human CRC cell lines (HCT116 and RKO) and a human normal colon epithelial cell line (CCD $841 \mathrm{CoN})$ were purchased from the ATCC. The cancer cell lines were cultured in DMEM, while CCD 841 CoN cells were cultured in EMEM. The media were supplemented with $10 \%$ heat-inactivated foetal bovine serum (FBS) and $1 \%$ antibiotics (100 U/ml penicillin). All cell lines were incubated at $37^{\circ} \mathrm{C}$ and maintained in an incubator with $5 \% \mathrm{CO}_{2}$. All cells were used while within a passage number that ranged between 8 and 21. Cells were seeded at 5,000 cells/well in 96-well plates for the WST-8 and bromodeoxyuridine (BrdU) assays, at 10,000 cells/well in 96-well plates for the lactate dehydrogenase $(\mathrm{LDH})$ release assay, at $2 \times 10^{5}$ cells/well in 6-well plates for the caspase-3/7 and Annexin V assays, and at $1 \times 10^{6}$ cells/dish in $6-\mathrm{cm}$ dishes for western blot analysis. Each cell line was seeded in the above culture media and incubated for $24 \mathrm{~h}$ prior to each experiment.

Evaluation of cell viability and proliferation. To evaluate the effects of TP4O on cell viability and proliferation, WST-8 and BrdU assays were performed. Briefly, cells were pre-incubated for $24 \mathrm{~h}$ and treated with various concentrations of TP4O for $24 \mathrm{~h}(0,1,10,100,1,000$ or $10,000 \mu \mathrm{M})$. Cell viability was quantified using a WST-8 assay via the Cell Counting kit-8 (Dojindo Molecular Technologies, Inc.) by measuring the absorbance at $450 \mathrm{~nm}$. Cell proliferation was quantified using a BrdU assay kit purchased from Roche Diagnostics $\mathrm{GmbH}$ by measuring the absorbance at $370 \mathrm{~nm}$. The half maximal inhibitory concentration $\left(\mathrm{IC}_{50}\right)$ values for each cell line were determined over $24 \mathrm{~h}$ following TP4O treatment. Cell viability following treatment with antioxidants was also measured. Following pre-incubation of the cells for $24 \mathrm{~h}$ at $37^{\circ} \mathrm{C}$, the culture medium was exchanged for medium with antioxidants (20 mM NAC, $20 \mu \mathrm{M}$ EB and $100 \mu \mathrm{M}$ MnTBAP) 30 min prior to TP4O administration. The results were obtained from three independent experiments.

Evaluation of apoptosis. To evaluate apoptosis, the following flow cytometry experiment was performed. After $24 \mathrm{~h}$ of pre-incubation at $37^{\circ} \mathrm{C}$, the cells were treated with various concentrations of $\mathrm{TP} 4 \mathrm{O}(0,100$ or $1,000 \mu \mathrm{M})$. Treatment-induced caspase-3/7 activation was examined in HCT116 and RKO cells using the Muse ${ }^{\mathrm{TM}}$ Cell Analyzer (Merck KGaA) and Muse ${ }^{\mathrm{TM}}$ Caspase-3/7 Assay kit (Merck $\mathrm{KGaA}$ ), according to the manufacturer's protocol. Following $6 \mathrm{~h}$ of treatment, harvested cells were mixed with the Muse $\mathrm{e}^{\mathrm{TM}}$ Caspase-3/7 reagent, which contains a DNA-binding dye that is linked to a DEVD peptide substrate and a dead cell marker [7-aminoactinomycin D (7-AAD)]. Caspase-3/7 activity was detected with the fluorescence of a DNA-binding dye from the Muse ${ }^{\mathrm{TM}}$ Caspase-3/7 Assay kit and cell viability was detected with 7-AAD fluorescence using the Muse ${ }^{\mathrm{TM}}$ Cell Analyzer. The results were obtained from three independent experiments.

For the Annexin V assay, cells were treated with TP4O $(0,100$ or $1,000 \mu \mathrm{M})$ for $12 \mathrm{~h}$. Treatment-induced apoptosis was examined using the Muse ${ }^{\mathrm{TM}}$ Annexin V \& Dead Cell kit (Merck KGaA) according to the manufacturer's protocol. Phosphatidylserine (PS) was detected using Annexin V, and cell viability was detected using a dead cell marker (7-AAD). The results were obtained from four independent experiments.

$L D H$-release assay. An LDH-release assay was performed to assess necrosis resulting from TP4O treatment. The LDH Cytotoxicity Detection kit (Takara Bio, Inc., Otsu, Japan) quantifies the activity of $\mathrm{LDH}$ released from damaged cells (18). Following $24 \mathrm{~h}$ of pre-incubation, the culture medium was exchanged for FBS-free DMEM containing 1\% Triton X-100, various concentrations of TP4O $(0,10,100$ or $1,000 \mu \mathrm{M}$ ) and $200 \mathrm{nM}$ staurosporine for $12 \mathrm{~h}$. Following treatment, the plates were centrifuged at $250 \mathrm{x} \mathrm{g}$ for $10 \mathrm{~min}$ at $4^{\circ} \mathrm{C}$. The supernatant was transferred to a clear 96-well plate, and $100 \mu \mathrm{l}$ of reaction mixture was then added to each well. The plates were incubated in the dark for $30 \mathrm{~min}$ at $25^{\circ} \mathrm{C}$, the absorbance was measured at $490 \mathrm{~nm}$ and the percentage $\mathrm{LDH}$ release was calculated. To compare necrotic cell death, samples with $1 \%$ Triton $\mathrm{X}-100$ were used as positive controls. To study apoptotic cell death, LDH release induced by $200 \mathrm{nM}$ staurosporine was examined. The results were obtained from three independent experiments.

Electron spin resonance (ESR) measurement. ROS generation in cells was measured using ESR, as previously described (19). Briefly, $10^{6}$ cells were seeded on a glass cover slide (49.0x5.0x0.2 $\mathrm{mm}$ ) and incubated overnight as previously described. Cells were treated with 0 or $1,000 \mu \mathrm{M}$ TP4O for $15 \mathrm{~min}$. ESR was measured in a respiration buffer containing 
$10 \mathrm{mM}$ DMPO as the spin-trapping agent. All ESR spectra were obtained using a JES-RE1X X-band spectrometer (JEOL, Ltd., Tokyo, Japan).

Detection of mitochondrial superoxide. The source of superoxide was detected by fluorescence microscopy. A total of $2.5 \times 10^{4}$ cells/well were seeded in an 8-well cover glass chamber. After $24 \mathrm{~h}$ of pre-incubation, cells were treated with 0 or $1,000 \mu \mathrm{M}$ TP4O for $24 \mathrm{~h}$. Culture medium was exchanged for phenol red-free DMEM, which contained $5 \mu \mathrm{M}$ MitoSOX Red $^{\text {TM }}$ Mitochondrial Superoxide Indicator (Molecular Probes; Thermo Fisher Scientific, Inc., Waltham, MA, USA), and the cells were incubated for $10 \mathrm{~min}$ at $37^{\circ} \mathrm{C}$. Following one wash with PBS, the cells were incubated for a further $30 \mathrm{~min}$ at $37^{\circ} \mathrm{C}$ with phenol-free DMEM, which contained $100 \mathrm{nM}$ MitoTracker Green FM ${ }^{\mathrm{TM}}$ (Molecular Probes; Thermo Fisher Scientific, Inc.). Next, the cells were washed with PBS and observed using a confocal inverted fluorescence microscope (Olympus Corporation, Tokyo, Japan). Localization of ROS derived from mitochondria was detected by MitoSOX Red ${ }^{\mathrm{TM}}$ Mitochondrial Superoxide Indicator fluorescence, and localization of mitochondria was detected by MitoTracker Green $\mathrm{FM}^{\mathrm{TM}}$ fluorescence. Images of bifocal field, MitoSOX Red ${ }^{\mathrm{TM}}$ Mitochondrial Superoxide Indicator and MitoTracker Green $\mathrm{FM}^{\mathrm{TM}}$ were merged.

Quantification of oxidative stress. The quantitative measurement of cellular populations undergoing oxidative stress was measured using the Muse ${ }^{\mathrm{TM}}$ Cell Analyzer and Muse ${ }^{\mathrm{TM}}$ Oxidative Stress kit (Merck KGaA). Cells were seeded at $2 \times 10^{5}$ cells/well in a 6 -well plate and incubated overnight. Following exposure to 0 or $1,000 \mu \mathrm{M}$ TP4O for $24 \mathrm{~h}$, according to the manufacturer's protocol, cells were detached, resuspended at $1 \times 10^{6}$ cells $/ \mathrm{ml}$ and incubated at $37^{\circ} \mathrm{C}$ for 30 min with Muse ${ }^{\text {TM }}$ Oxidative Stress working solution, which contained dihydroethidium (DHE). DHE is cell permeable, and has been proposed to react with superoxide anions, thus undergoing oxidation upon binding to DNA (20). The number of oxidized cells were counted according to the intensity of red fluorescence using the Muse ${ }^{\mathrm{TM}}$ Cell Analyzer. The results were obtained from four independent experiments.

Western blot analysis. Cells were seeded at $1 \times 10^{6}$ cells/dish in 6-cm dishes. After $24 \mathrm{~h}$, cells were treated with various concentrations of $\operatorname{TP} 4 \mathrm{O}(0,100$ or $1,000 \mu \mathrm{M})$ for $24 \mathrm{~h}$. Cultured cells were washed with PBS and lysed with lysis buffer that contained 1\% Protease Inhibitor Cocktail (100X; Cell Signaling Technology, Inc., Danvers, MA, USA) and $1 \mathrm{mM}$ phenylmethylsulfonyl fluoride. Samples were kept on ice for $2 \mathrm{~min}$, followed by sonication for $5 \mathrm{~min}$ and centrifugation at $13,000 \mathrm{x}$ g for $2 \mathrm{~min}$ at $4^{\circ} \mathrm{C}$; the supernatants were then collected. The samples were subjected to $15 \%$ SDS-PAGE and transferred to a polyvinylidene fluoride membrane (Merck $\mathrm{KGaA}$ ). After 30 min blocking with Tris-buffered saline containing $0.1 \%$ Tween-20 (T-TBS) and 5\% bovine serum albumin (Iwai Chemicals Company, Ltd., Tokyo, Japan), primary antibodies against superoxide dismutase 2 (SOD2; cat. no. 13141; dilution, 1:1,000) glutathione peroxidase 1 (GPX1; cat. no.3206; dilution 1:500) and GAPDH (endogenous control; cat. no. 2118; dilution, 1:1,000; all from Cell Signalling
Technology, Inc.) were incubated with membranes in Can Get Signal ${ }^{\circledR}$ Immunoreaction Enhancer Solution 1 (Toyobo Co., Ltd., Osaka, Japan) overnight at $25^{\circ} \mathrm{C}$. After washing 3 times for 30 min with T-TBS, the membrane was incubated with an anti-rabbit IgG, horseradish perxodiase-linked antibody (cat. no. 7074; dilution, 1:1,000; Cell Signalling Technology, Inc.) in Can Get Signal ${ }^{\circledR}$ Immunoreaction Enhancer Solution 2 (Toyobo Co., Ltd.) for $2 \mathrm{~h}$ at $25^{\circ} \mathrm{C}$. After washing 3 times for 15 min with T-TBS at $25^{\circ} \mathrm{C}$, the bands were incubated with ECL $^{\mathrm{TM}}$ Western Blotting Detection reagents as according to the manufacturer's protocol and detected with ImageQuant LAS 4000 mini (both from GE Healthcare Life Sciences, Chalfont, UK). Results were quantified using ImageQuant TL 7.0 software (GE Healthcare Life Sciences).

Animals. A total of 14 male ICR-SCID mice aged 6-7 weeks old and weighing 21-25 g were purchased from Charles River Laboratories Japan, Inc. (Yokohama, Japan). The mice were provided with clean water and food ad libitum, and housed in standardized, pathogen-free conditions with a $14 \mathrm{~h} \mathrm{light} / 10 \mathrm{~h}$ dark cycle, a temperature of $23.5 \pm 2.5^{\circ} \mathrm{C}$ and humidity of $52.5 \pm 12.5 \%$. The mice were used after an acclimation period of $\geq 7$ days. All animal experiments were performed with the approval of the Animal Ethics Committee of the University of Tsukuba (Tsukuba, Japan; approval no. 13-385) and according to the guidelines of this committee.

Xenograft model. To evaluate the effect of TP4O in vivo, a subcutaneous tumor was induced. HCT116 cells $\left(2 \times 10^{6}\right.$ cells per mouse) were injected subcutaneously into the right flanks of 14 mice. Tumor volume was calculated using the following formula: $0.5 \mathrm{x}$ length $\mathrm{x}$ width ${ }^{2}$. When tumor volume had reached $80-100 \mathrm{~mm}^{3}$, the 12 qualifying mice were randomly divided into two groups (n=6/group) and subcutaneously injected with one of the following: $250 \mu \mathrm{l}$ saline (control group) or $200 \mathrm{mg} / \mathrm{kg}$ TP4O dissolved in $250 \mu \mathrm{l}$ saline (TP4O group). Each mouse was injected once every 3 days (total, five times). The tumor volume was recorded every 3 days. Body weight was recorded the first day of the injection, on day 7 and at the end of experimentation (day 14). On day 14, blood samples were collected under anesthesia, the mice were sacrificed and the tumors were excized. To evaluate TP4O toxicity, serum alanine aminotransferase (ALT) and serum creatinine levels were measured using an autoanalyzer (Dri-chem $7000 \mathrm{~V}$; Fujifilm, Tokyo, Japan).

Immunostaining. The tumor tissue was fixed with $10 \%$ formaldehyde. Each $2-\mu \mathrm{m}$ paraffin-embedded section was stained with an anti-8-hydroxy-2'-deoxyguanosine (8-OHdG) antibody at $4^{\circ} \mathrm{C}$ overnight (cat. no. MOG-20P; dilution, 1:100, Japan Institute for the Control of Aging, Shizuoka, Japan) or with an anti-cleaved caspase-3 antibody according to the manufacturer's protocol (cat. no. 9661; dilution, 1:300; Cell Signaling Technology, Inc.). 8-OHdG is a marker for oxidative stress (21). To accurately quantify $8-\mathrm{OHdG}$ - and cleaved caspase-3-positive areas, slides from three randomly selected high-power fields (magnification, x200) of each tumor were visualized using a BZ-X710 microscope (Keyence Corporation, Osaka, Japan). An automated software analysis program, BZ-X analyser version 1.3.0.3 (Keyence Corporation), was 
A

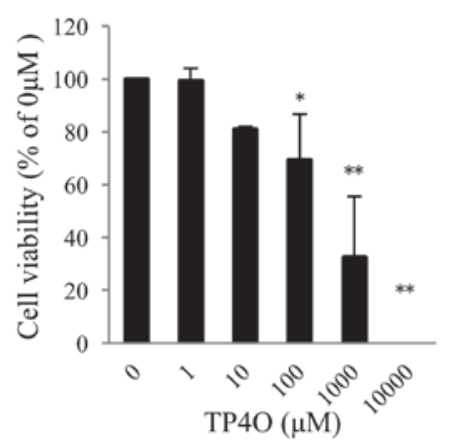

HCT116

$\mathrm{B}$

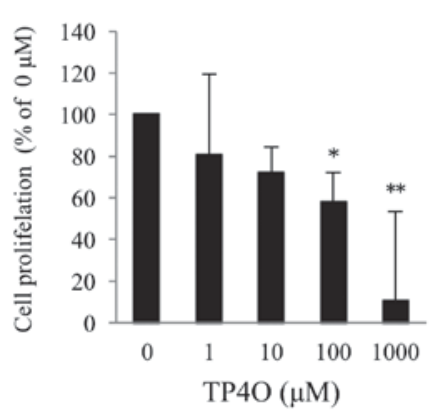

HCT116

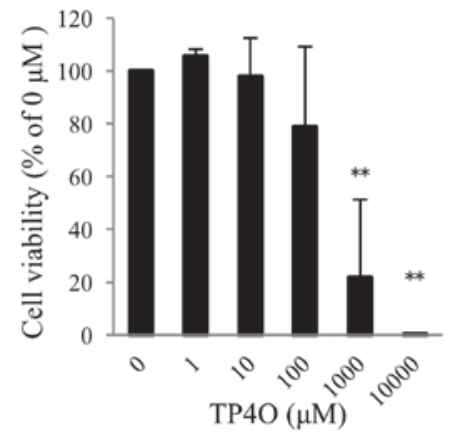

RKO

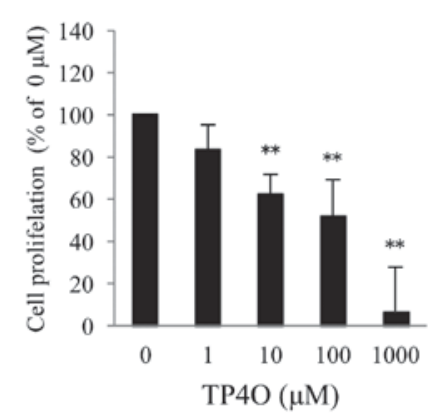

RKO

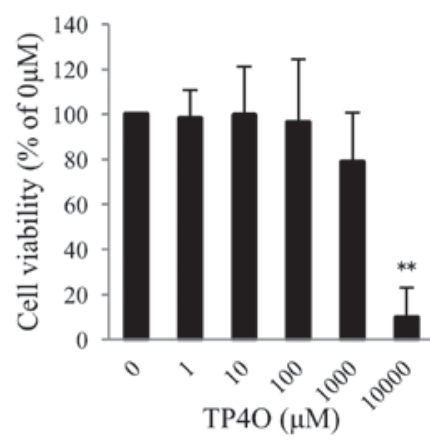

CCD841CoN

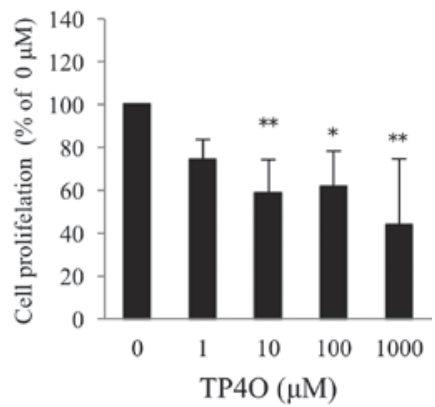

CCD841CoN

Figure 1. TP4O causes cell death and inhibits cell proliferation in human CRC cell lines. (A) Cell viability was measured using the WST-8 assay. HCT116, RKO and CCD $841 \mathrm{CoN}$ cells were treated with the indicated concentrations of TP4O for $24 \mathrm{~h}$. Values were compared with $0 \mu \mathrm{M}$ TP4O as the control, which was considered $100 \%$. Cell viability was decreased in CRC cells in a dose-dependent manner. The $\mathrm{IC}_{50}$ values of TP4O in HCT116 and RKO cells were 661 and $381 \mu \mathrm{M}$, respectively. The $\mathrm{IC}_{50}$ value of TP4O in the human normal colon epithelial cell line CCD $841 \mathrm{CoN}$ was 5,347 $\mu \mathrm{M}$. (B) Cell proliferation was measured using a bromodeoxyuridine assay. HCT116, RKO and CCD $841 \mathrm{CoN}$ cells were treated with the indicated concentrations of TP4O for $24 \mathrm{~h}$. The values were compared with those obtained with $0 \mu \mathrm{M}$ TP4O, which served as the control (100\%). The percentage of cell proliferation decreased in a dose-dependent manner. The data are represented as the mean \pm standard deviation. ${ }^{~} \mathrm{P}<0.05 .{ }^{* *} \mathrm{P}<0.01$. The results were obtained from three independent experiments. TP4O, terpinen-4-ol; CRC, colorectal cancer; $\mathrm{IC}_{50}$, half maximal inhibitory concentration.

used to determine the percentage of stained areas in the digital photomicrographs.

Statistical analysis. The data are expressed as the mean \pm standard deviation. For comparison of $>2$ groups, analysis of variance (ANOVA) was performed. If the ANOVA results were significant, a Dunnett's test for comparison with the control was used. For comparison of two groups, an unpaired Student's t-test was performed. All indicated P-values were two-sided, and $\mathrm{P}<0.05$ was considered to indicate a statistically significant difference. All statistical analyses were performed using GraphPad Prism software version 5.01 (GraphPad Software, Inc., La Jolla, CA, USA).

\section{Results}

TP4O inhibits CRC cell viability and proliferation. To evaluate cytotoxicity following TP4O treatment, cell viability was measured using a WST-8 assay. TP4O significantly decreased the viability of the human CRC cell lines HCT116 at 100, 1,000 and 10,000 $\mu \mathrm{M}$, and RKO at 1,000 and 10,000 $\mu \mathrm{M}$ (Fig. 1A). The effect of TP4O was dose dependent. The $\mathrm{IC}_{50}$ values of TP4O in HCT116 and RKO cells were 661 and $381 \mu \mathrm{M}$, respectively. By contrast, the $\mathrm{IC}_{50}$ value of TP4O in the normal human colon epithelial cell line CCD $841 \mathrm{CoN}$ was $5,347 \mu \mathrm{M}$.
In addition, TP4O significantly inhibited cell proliferation in a dose-dependent manner in CRC cell lines and CCD 841 CoN cell line, particularly in the CRC cell lines at $1,000 \mu \mathrm{M}$ (Fig. 1B).

TP4O induces apoptotic cell death in CRC cells. TP4O-induced apoptotic cell death was determined using a caspase-3/7 activity assay and Annexin V assay. DNA fragmentation and exposure of PS are typical phenomena observed in apoptotic cell death (22). Caspase-3/7 activity was enhanced in TP4O-treated cells in a dose-dependent manner (Fig. 2A). The distribution of apoptotic cells, which were located in the top- and bottom-right quadrants, is presented in a bar graph; the percentage of apoptotic cells was significantly increased in cells treated with $1,000 \mu \mathrm{M}$ TP4O compared with $0 \mu \mathrm{M}$ TP4O $(\mathrm{P}<0.05$; Fig. 2B). Although there was no statistical difference, the percentage of Annexin $\mathrm{V}$-positive cells tended to increase in HCT116 and RKO cell cultures treated with 1,000 $\mu \mathrm{M} \mathrm{TP} 4 \mathrm{O}$ (Fig. 2C and D). The lower level of LDH release following TP4O treatment indicates that TP4O did not induce necrotic LDH release in HCT116, RKO (Fig. 2E) or CCD 841 CoN (data not shown) cell lines.

TP4O generates ROS in CRC cells, and TP4O-induced cell death is rescued by antioxidant reagents. In the presence of 

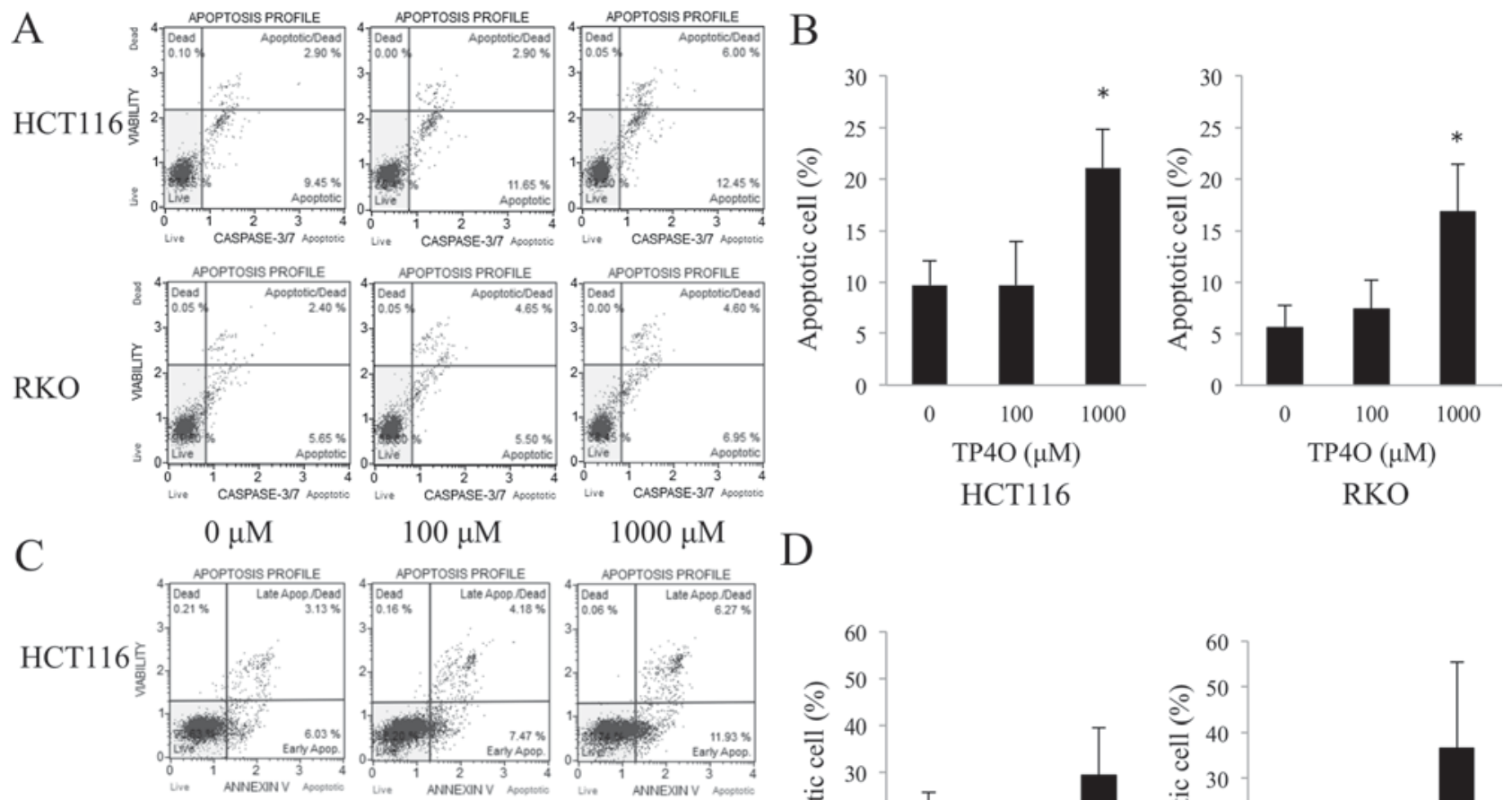

$\mathrm{D}$

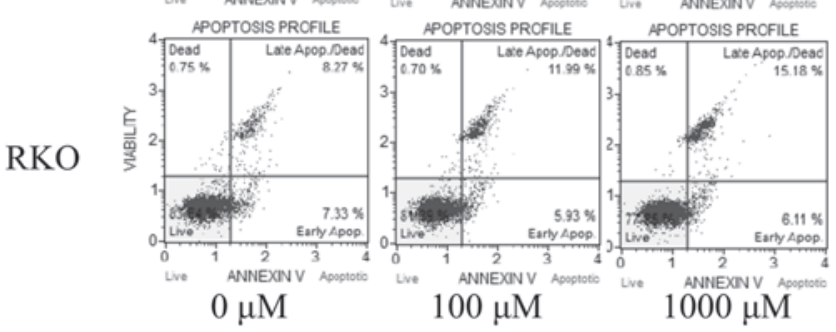

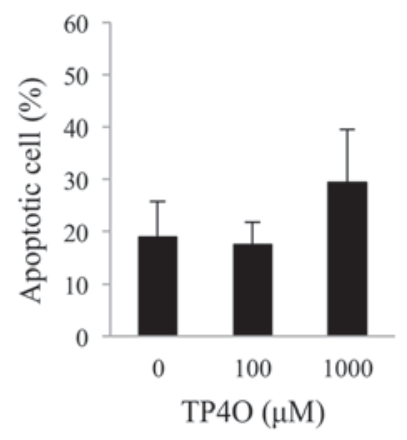

HCT116

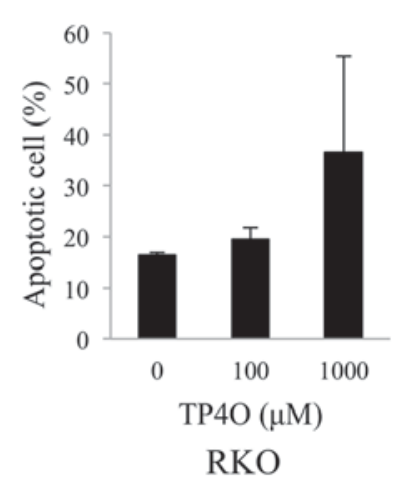

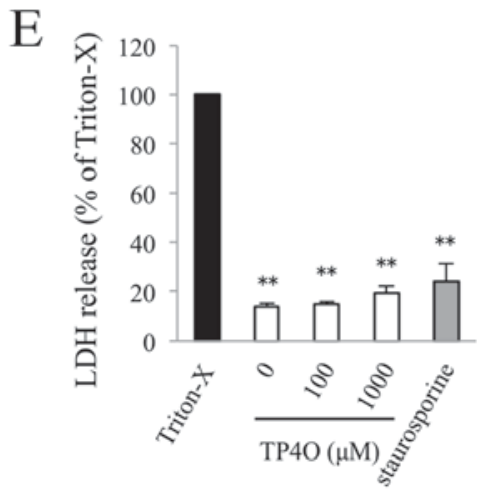

HCT116

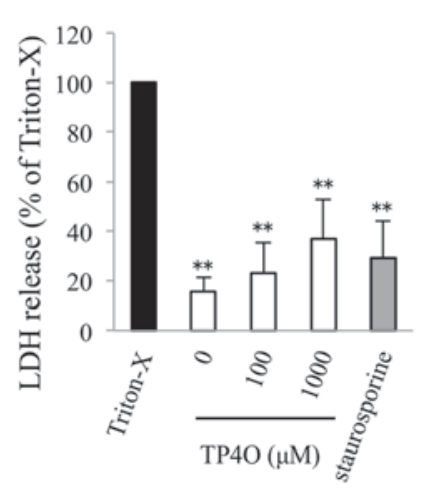

RKO

Figure 2. TP4O induces the apoptosis of human CRC cell lines. (A) HCT116 and RKO cells were treated with the indicated concentrations of TP4O for $6 \mathrm{~h}$. Caspase-3/7 activity and cell viability were measured by flow cytometry. Caspase-3/7 activity was detected by fluorescence of a DNA-binding dye and cell viability were detected with the detection of 7-aminoactinomycin D fluorescence using the Muse ${ }^{\mathrm{TM}}$ Cell Analyzer. (B) The percentage of apoptotic cells, which are located in the top- and bottom-right quadrants, is presented. The values were compared with those obtained with $0 \mu \mathrm{M} \mathrm{TP} 4 \mathrm{O}$. The data are presented as the mean $\pm \mathrm{SD}$. ${ }^{*} \mathrm{P}<0.05$. The results were obtained from three independent experiments. (C) HCT116 and RKO cells were treated with the indicated concentrations of TP4O for $12 \mathrm{~h}$. The extent of apoptosis was measured with Annexin V staining. (D) The percentage of apoptotic cells, which are located in the top- and bottom-right quadrants, is presented. The values were compared to with those obtained with $0 \mu \mathrm{M}$ TP4O. The data are presented as the mean \pm SD. The results were obtained from four independent experiments. (E) HCT116 and RKO cells were treated with the indicated concentrations of TP4O for $12 \mathrm{~h}$, and the LDH level in the supernatant was measured. The values were compared with those obtained with $1 \%$ Triton X-100 as the control (100\%). To determine whether LDH release was associated with necrosis or apoptosis, cells were treated with Triton X-100 or staurosporine, respectively. The data are represented as the mean $\pm \mathrm{SD} .{ }^{* * *} \mathrm{P}<0.01$. The results were obtained from three independent experiments. TP4O, terpinen-4-ol; CRC, colorectal cancer; IC ${ }_{50}$, half maximal inhibitory concentration; SD, standard deviation; LDH, lactate dehydrogenase; Apop., apoptosis.

DMPO, the hydroxyl radical can be detected as as four spikes in ESR (23). ESR measurements revealed that treatment with $1,000 \mu \mathrm{M}$ TP4O for 15 min induced the generation of the hydroxyl radical in CRC cells (Fig. 3A), whereas the hydroxyl radical was barely detectable in CCD841 CoN cells (data not shown). The increase in oxidative stress was quantified by DHE reaction. TP4O administration led to an increase in the number of cells with high ROS (Fig. 3B). There was a significant increase in oxidative stress in HCT116 cells $(\mathrm{P}<0.05$; Fig. $3 \mathrm{C})$. This result implies that TP4O enhances the accumulation of intracellular ROS in HCT116 cells. There was a non-significant increase observed in RKO cells following the administration of TP4O. 
A $0 \mu \mathrm{M}$
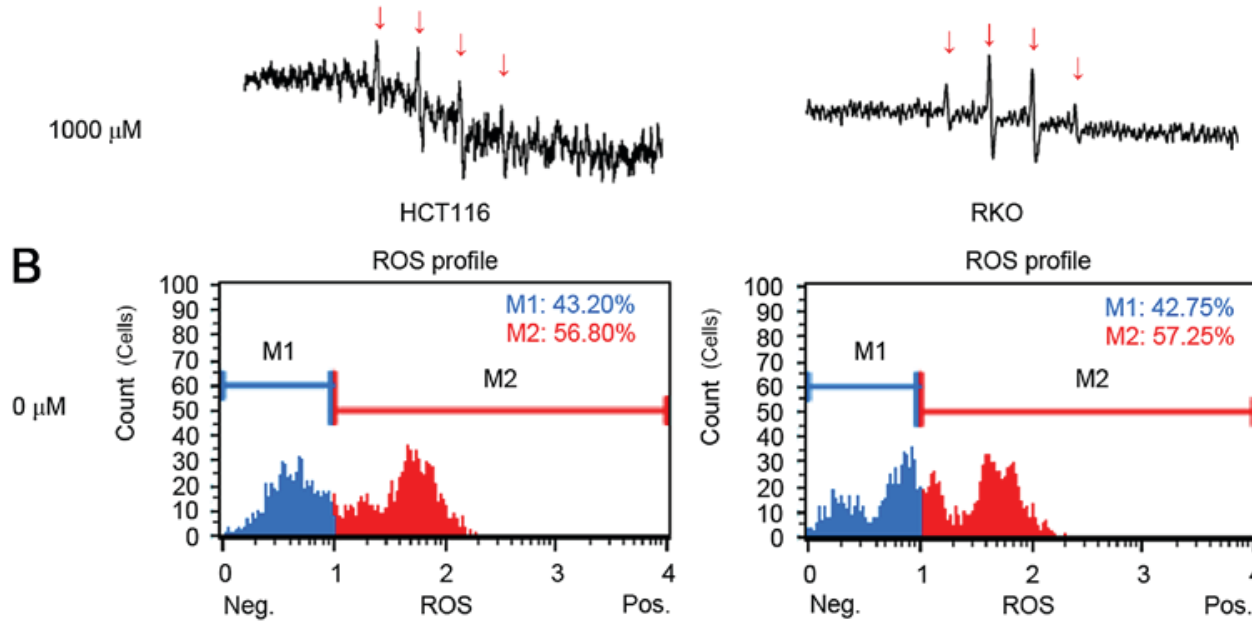

RKO
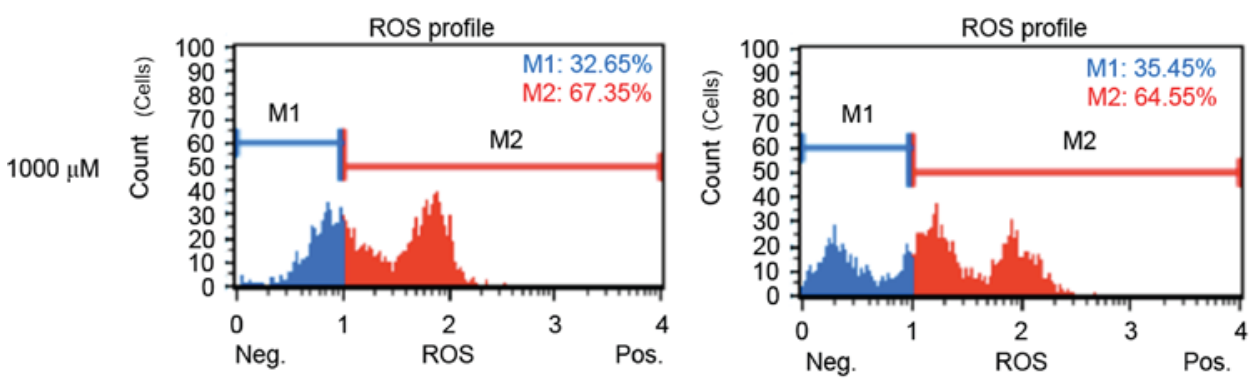

HCT116

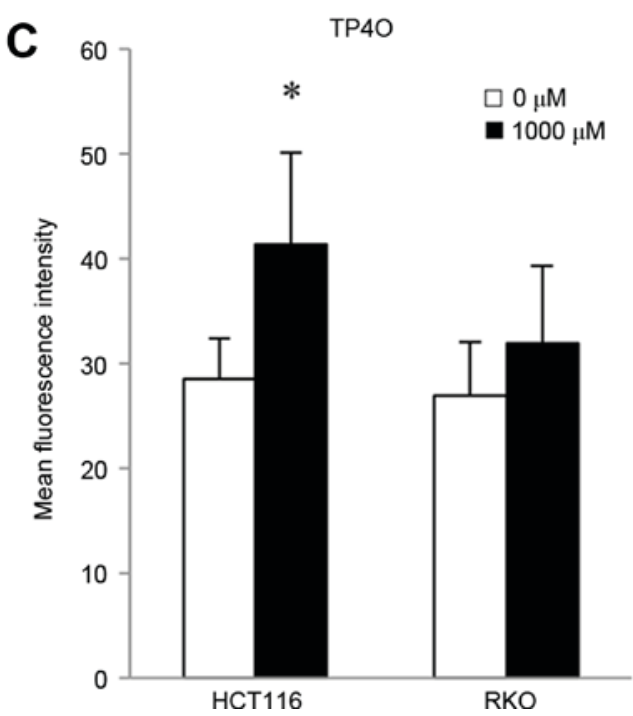

Figure 3. TP4O induces ROS in human CRC cell lines. (A) ESR measurement. HCT116 and RKO cells were treated with 0 or $1,000 \mu \mathrm{M}$ of TP4O for 15 min. ESR was measured in a respiration buffer containing 5,5-dimethyl-1-pyrroline-N-oxide as the spin-trapping agent. The production of the hydroxyl radical was detected with four spikes in ESR. Spikes derived from the hydroxyl radical (indicated with arrows) became apparent in cells exposed to 1,000 $\mu \mathrm{M}$ TP4O. (B) Quantitative measurements of cellular populations undergoing oxidative stress, based on the detection of ROS. HCT116 and RKO cells were untreated or treated with 1,000 $\mu \mathrm{M} \mathrm{TP} 4 \mathrm{O}$ for $24 \mathrm{~h}$. The distribution of cells according to levels of intracellular ROS was represented as a histogram. Cells were divided into two groups: The blue area (M1) represented the cells with low ROS, and the red area (M2) represented the cells with high ROS. The administration of TP4O led to an increase in the number of cells in the red area. (C) The mean fluorescence intensity of each group is presented. The values were compared with those obtained with $0 \mu \mathrm{M}$ TP4O. The data are presented as the mean $\pm \mathrm{SD}$. ${ }^{*} \mathrm{P}<0.05$. The results were obtained from four independent experiments. $\mathrm{TP} 4 \mathrm{O}$, terpinen-4-ol; CRC, colorectal cancer; $\mathrm{IC}_{50}$, half maximal inhibitory concentration; SD, standard deviation; ROS, reactive oxygen species; DHE, dihydroethidium; ESR, electron spin resonance.

To determine the source of TP4O-induced ROS, CRC cells were observed by confocal inverted fluorescence microscopy. The location of the mitochondria was represented by
MitoTracker Green $\mathrm{FM}^{\mathrm{TM}}$ fluorescence, while accumulated ROS were represented by MitoSOX Red ${ }^{\mathrm{TM}}$ Mitochondrial Superoxide Indicator fluorescence. The merged image 


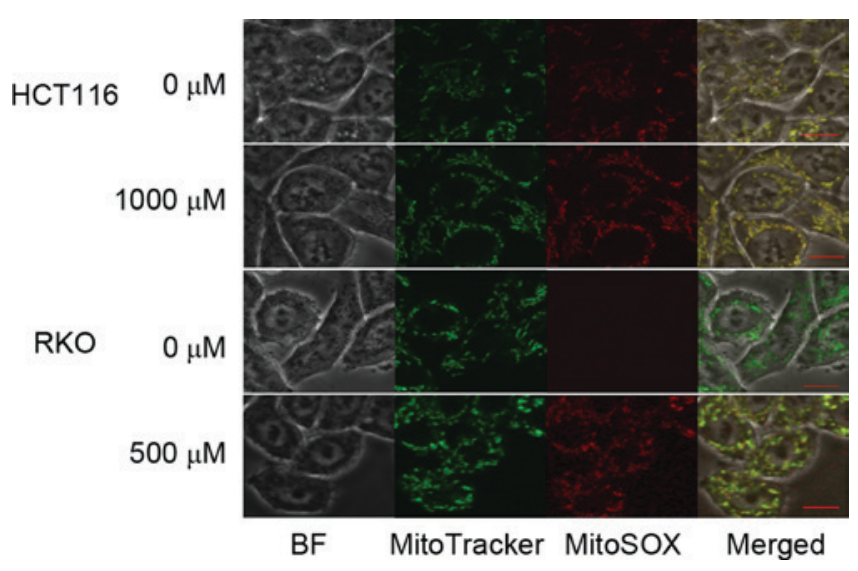

Figure 4. Localization of ROS using MitoSOX Red ${ }^{\mathrm{TM}}$ Mitochondrial Superoxide Indicator fluorescence in HCT116 and RKO cells. HCT116 and RKO cells were treated with the indicated concentration of TP4O for $24 \mathrm{~h}$. To locate the mitochondria generating ROS, the same cells were incubated with MitoTracker Green FM ${ }^{\mathrm{TM}}$ and MitoSOX Red ${ }^{\mathrm{TM}}$ Mitochondrial Superoxide Indicator. Images of merged MitoTracker Green FM ${ }^{\mathrm{TM}}$ and MitoSOX Red ${ }^{\mathrm{TM}}$ Mitochondrial Superoxide Indicator fluorescence were constructed to identify ROS generated by mitochondria. Scale bars represent $10 \mu \mathrm{m}$. ROS reactive oxygen species; TP4O, terpinen-4-ol.

demonstrated that the ROS induced by TP4O were generated by mitochondria (Fig. 4).

Protein levels of intrinsic oxidant scavengers were analysed by western blotting. Protein levels of SOD2 and GPX1 tended to increase following exposure to TP4O (Fig. 5A). However, there was no significant difference in comparison with that of $0 \mu \mathrm{M}$ TP4O (Fig. 5B). The results suggested that ROS accumulation was not due to suppression of ROS scavengers, but due to increased ROS production. CRC cells were exposed to specific antioxidants (NAC, EB and MnTBAP), which prevented significant TP4O-derived cell death (Fig. 5C), demonstrating that ROS accumulation was the cause for cell death.

TP4O treatment inhibits tumor growth in a xenograft mouse model. A schematic representation of the experimental design is provided in Fig. 6A. Mice treated with TP4O developed significantly smaller tumors compared with the group treated with vehicle $(\mathrm{P}<0.05$; Fig. 6B). TP4O administration did not cause marked complications or body weight loss, and the serum levels of ALT and creatinine were not different in the TP4O group compared with those in the control group (Fig. 6C). For the histological analysis, tumors were stained using antibodies against the oxidative stress marker $8-\mathrm{OHdG}$. The areas of anti-8-OH-dG staining were larger in tissues from the TP4O group compared with those from the control group (Fig. 6D). In addition, areas stained by anti-cleaved caspase-3 antibody were larger in the TP4O group than in the control group (Fig. 6D).

\section{Discussion}

TP4O is an essential oil extracted from aromatic plants and is a type of monoterpene (10). Monoterpenes, the C10 class of isoprenoids, are a diverse family of natural products, ranging in structure from linear to polycyclic (24). Menthol, limonene, eucalyptol and citral are familiar monoterpenes that are used as food and cosmetic additives $(8,24)$.
The anticancer effects of essential oils have previously been investigated by biological approaches focusing on pharmaceutical and therapeutic potentials of essential oils $(7,9,10,25)$. Previous studies have indicated that the anticancer effects of TPO4 are due to the induction of apoptosis in human melanoma cells (14), human non-small cell lung cancer (15), human leukaemia cells (16) and CRC cells (17). However, Greay et al (26) reported that TP4O induces necrosis and cell cycle arrest in murine mesothelioma and melanoma cells. In the present study, TP4O elevated caspase-3/7 activity and increased the number of Annexin V-positive cells without inducing necrotic LDH release in CRC cells. In the in vivo experiments, the number of cleaved caspase-3-positive cells increased significantly in the TP4O group compared with that in the control group. These results indicated that TP4O induced cell death in human CRC cell lines by apoptosis in vitro and in vivo.

In addition, the data from the present study revealed the source of ROS generation in the in vitro and in vivo TP4O treatments. Previous studies have demonstrated the antioxidant activity of essential oils [reviewed in (7)], including TTO, which contains TP4O (27). Kim et al (28) investigated the antioxidant activity of TTO, and determined that three compounds of TTO serve important roles in the antioxidant mechanism, whereas TP4O did not possess antioxidant activity. In the current study, the hydroxyl radical was directly detected by ESR. Moreover, the protein levels of the major ROS scavengers and GPX1 were not decreased, which indicates that the increase in ROS following TP4O treatment is not due to inhibition of the redox system but is the result of an increase in ROS production. The addition of antioxidant reagents inhibited TP4O-induced cell death. Taken together, these results suggested that the ROS induced following TP4O exposure serve a role in the anticancer effect of TP4O.

To maintain active proliferation, cancer cells produce more oxidative metabolites than normal cells (29). The redox system is activated in cancer, but cancer cells are not able to adapt to excess oxidative stress due to a decreased capacity for ROS metabolism compared with that of normal cells (29). However, normal cells produce lower levels of ROS than cancer cells; thus, they can adapt to increases in $\operatorname{ROS}(30,31)$. Previous reports have demonstrated that the differences in cellular metabolism of ROS could be a target for cancer treatment (31-37). There are three strategies to amplify intracellular oxidative stress. The first one is the inhibition of redox enzymes, i.e., GPX (35) or SOD (31). The second one is facilitating ROS production (36); the use of TP4O is included in this category. The third strategy is a combination of the two strategies mentioned above (37). These previous studies have revealed $\mathrm{IC}_{50}$ discrepancies between cancer cells and normal cells. The results of the present study support those from previous reports suggesting that ROS-mediated compounds have therapeutic potential against malignant neoplasms.

A number of limitations of the present study should be acknowledged. First, the underlying molecular mechanisms of ROS production by TP4O or the apoptotic pathway involved have not been detailed. As indicated by previous reports, excessive production and accumulation of ROS affects several mitogen-activated protein kinase signalling pathways, e.g., p38 mitogen-activated protein kinase, extracellular signal-regulated 
A

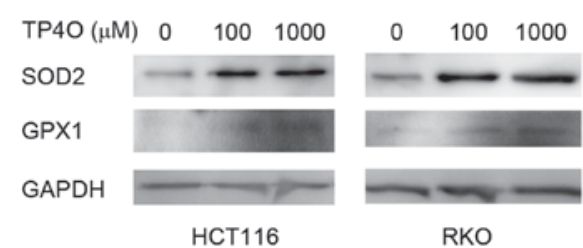

C

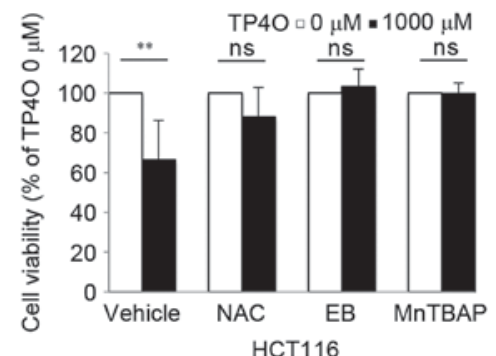

B TP4O $\square 0 \mu \mathrm{M}$
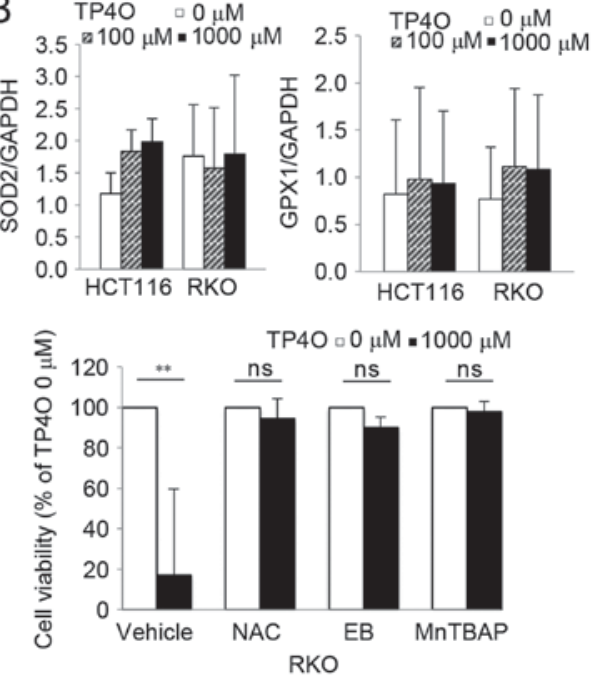

Figure 5. TP4O induces the activation of antioxidants, and antioxidant reagents rescue TP4O cytotoxicity. (A) Western blot analysis of antioxidant proteins Lysates of HCT116 and RKO cells were examined following treatment with the indicated concentrations of TP4O for $24 \mathrm{~h}$. GAPDH was used as a loading control. (B) HCT116 and RKO cells were treated with 0 or $1,000 \mu \mathrm{M}$ TP $4 \mathrm{O}$ in the presence of vehicle, $20 \mathrm{mM} \mathrm{NAC}, 20 \mu \mathrm{M} \mathrm{EB}$ or $100 \mu \mathrm{M}$ MnTBAP for $24 \mathrm{~h}$, and cell viability was measured by WST- 8 assay. The values for each group were compared with those obtained with $0 \mu \mathrm{M}$ TP4O. The data are represented as the mean \pm standard deviation. ${ }^{* *} \mathrm{P}<0.01$. The results were obtained from three independent experiments. TP4O, terpinen-4-ol; MnTBAP, manganese (III) tetrakis (4-benzoic acid) porphyrin chloride; SOD2, superoxide dismutase 2; GPX1, glutathione peroxidase 1; NAC, N-acetyl-L-cysteine; EB, ebselen; ns, not significant.

A
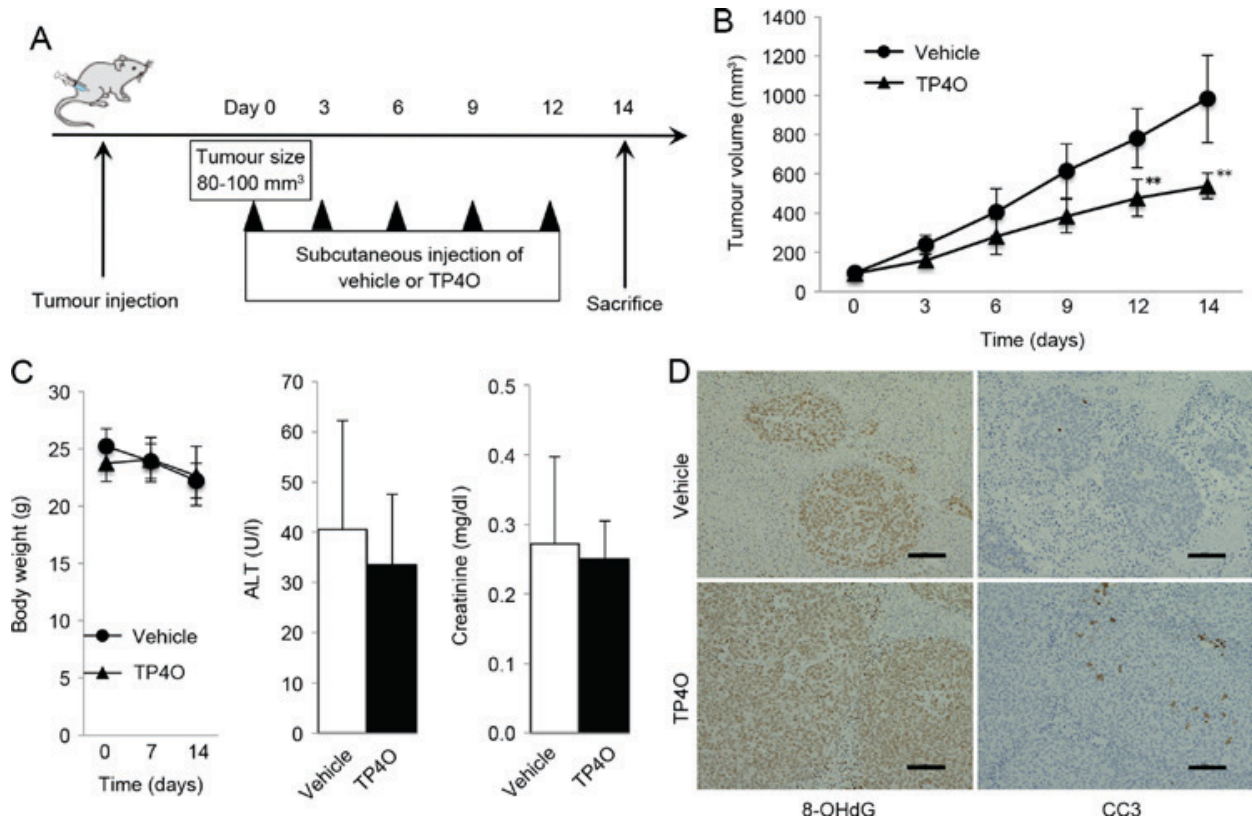

Figure 6. In vivo antitumor activity of TP4O in a xenograft model. (A) Schematic experimental design. A total of $2 \times 10^{6} \mathrm{HCT} 116$ cells were subcutaneously injected into the right flank of ICR-SCID mice receiving $250 \mu 1$ of vehicle or TP4O $(200 \mathrm{mg} / \mathrm{kg})$ every 3 days by subcutaneous injection. Mice were sacrificed 14 days after the first day of administration. (B) Tumor volume was measured over time. Increase in tumor volume was suppressed in the TP4O group. The data are presented as the mean \pm standard deviation. ${ }^{* *} \mathrm{P}<0.01$. (C) Mice were sacrificed after 14 days, and body weight as well as serum levels of ALT and creatinine were measured. (D) Tumors were stained with antibodies against 8-OHdG and CC3. 8-OHdG- and CC3-stained positive areas were assessed by the ratio of the stained area to the entire tumor area in high-magnification (x200) digital images in three randomly selected fields for each sample. Scale bars represent $100 \mu \mathrm{m}$. TP4O, terpinen-4-ol; CC3, cleaved caspase-3; 8-OHdG, 8-hydroxy-2'-deoxyguanosine; ALT, alanine aminotransferase.

kinase and c-Jun N-terminal kinase signalling pathways, which may be associated with ROS production $(31,34,38,39)$. Thus, further studies are required to elucidate the association between TP4O and ROS generation or signalling pathways. Second, the activities of ROS scavengers were estimated using western blot analysis of SOD2 and GPX1; however, the actual activities of these enzymes or other redox systems have not been measured.
The possibility that TP4O suppresses other redox systems cannot be ruled out. Third, ROS cause DNA damage and can promote tumorigenic signalling pathways (40). ROS are also known to lead to Alzheimer's disease (41), ischaemic vascular disease (42), non-alcoholic steatohepatitis (43) and other diseases (44). Although the data from the current study demonstrated that TP4O inhibited CRC xenograft growth without marked liver 
or renal injury in vivo, more precise assessment of the risk of the use of ROS for cancer therapy is required. The therapeutic concentration in the present study of TPO4 was similar to that of a previous report (17), which is considered high in clinical use. Therefore, consideration of a practical dose for future clinical use is crucial. As Shapira et al (17) reported, combination therapy may reduce the dose of TP4O and existing cancer drugs. Additionally, Berndtsson et al (45) reported the ROS accumulation effect of oxaliplatin, which is typically used for the treatment of CRC (4). Thus, it is expected that combination therapy with TP4O and oxaliplatin would result in a synergistic effect. Upon sufficiently addressing the aforementioned issues, this new therapeutic strategy could be applicable in a clinical setting.

In conclusion, the results from the present study suggest that TP4O induces apoptosis in CRC cells through the generation of ROS in vitro and in vivo without damaging normal cells. Furthermore, TP4O is potentially useful for the future development of therapies against CRC.

\section{Acknowledgements}

The authors thank Dr Yumiko N. Nagano (Department of Gastroenterology) for her technical advice on evaluating ROS and Mrs. Ako Takahashi (Department of Surgery, Division of Gastroenterological and Hepatobiliary Surgery and Organ Transplantation, Faculty of Medicine, University of Tsukuba) for her technical assistance. The authors also thank the American Journal Experts (Durham, NC, USA) for proofreading their English writing. The present study was supported in part by a grant from the Ministry of Education, Culture, Sports, Science and Technology of Japan (Grants-in-Aid for Scientific Research; grant no. 25462069).

\section{References}

1. Torre LA, Bray F, Siegel RL, Ferlay J, Lortet-Tieulent J and Jemal A: Global cancer statistics, 2012. CA Cancer J Clin 65: 87-108, 2015.

2. DeSantis CE, Lin CC, Mariotto AB, Siegel RL, Stein KD, Kramer JL, Alteri R, Robbins AS and Jemal A: Cancer treatment and survivorship statistics, 2014. CA Cancer J Clin 64: 252-271, 2014.

3. Scott AM, Wolchok JD and Old LJ: Antibody therapy of cancer. Nat Rev Cancer 12: 278-287, 2012.

4. Gustavsson B, Carlsson G, Machover D, Petrelli N, Roth A, Schmoll HJ, Tveit KM and Gibson F: A review of the evolution of systemic chemotherapy in the management of colorectal cancer. Clin Colorectal Cancer 14: 1-10, 2015.

5. Mann J: Natural products in cancer chemotherapy: Past, present and future. Nat Rev Cancer 2: 143-148, 2002.

6. Newman DJ and Cragg GM: Natural products as sources of new drugs over the 30 years from 1981 to 2010. J Nat Prod 75: 311-335, 2012.

7. Edris AE: Pharmaceutical and therapeutic potentials of essential oils and their individual volatile constituents: A review. Phytother Res 21: 308-323, 2007.

8. Aggarwal BB, Prasad S, Reuter S, Kannappan R, Yadev VR, Park B, Kim JH, Gupta SC, Phromnoi K, Sundaram VR, et al: Identification of novel anti-inflammatory agents from Ayurvedic medicine for prevention of chronic diseases: 'Reverse pharmacology' and 'bedside to bench' approach. Curr Drug Targets 12: $1595-1653,2011$.

9. Gautam N, Mantha AK and Mittal S: Essential oils and their constituents as anticancer agents: A mechanistic view. Biomed Res Int 2014: 154106, 2014.

10. Sobral MV, Xavier AL, Lima TC and de Sousa DP Antitumor activity of monoterpenes found in essential oils. ScientificWorldJournal 2014: 953451, 2014.
11. Pazyar N, Yaghoobi R, Bagherani N and Kazerouni A: A review of applications of tea tree oil in dermatology. Int J Dermatol 52: 784-790, 2013.

12. Carson CF and Riley TV: Antimicrobial activity of the major components of the essential oil of Melaleuca alternifolia. J Appl Bacteriol 78: 264-269, 1995.

13. Hart PH, Brand C, Carson CF, Riley TV, Prager RH and Finlay-Jones JJ: Terpinen-4-ol, the main component of the essential oil of Melaleuca alternifolia (tea tree oil), suppresses inflammatory mediator production by activated human monocytes. Inflamm Res 49: 619-626, 2000.

14. Calcabrini A, Stringaro A, Toccacieli L, Meschini S, Marra M, Colone M, Salvatore G, Mondello F, Arancia G and Molinari A: Terpinen-4-ol, the main component of Melaleuca alternifolia (tea tree) oil inhibits the in vitro growth of human melanoma cells. J Invest Dermatol 122: 349-360, 2004.

15. Wu CS, Chen YJ, Chen JJ, Shieh JJ, Huang CH, Lin PS, Chang GC, Chang JT and Lin CC: Terpinen-4-ol induces apoptosis in human nonsmall cell lung cancer in vitro and in vivo. Evid Based Complement Alternat Med 2012: 818261, 2012.

16. Banjerdpongchai R and Khaw-On P: Terpinen-4-ol induces autophagic and apoptotic cell death in human leukemic HL-60 cells. Asian Pac J Cancer Prev 14: 7537-7542, 2013.

17. Shapira S, Pleban S, Kazanov D, Tirosh P and Arber N: Terpinen-4-ol: A novel and promising therapeutic agent for human gastrointestinal cancers. PLoS One 11: e0156540, 2016.

18. Legrand C, Bour JM, Jacob C, Capiaumont J, Martial A, Marc A, Wudtke M, Kretzmer G, Demangel C, Duval D, et al: Lactate dehydrogenase (LDH) activity of the cultured eukaryotic cells as marker of the number of dead cells in the medium [corrected]. J Biotechnol 25: 231-243, 1992.

19. Tamura M, Matsui H, Kaneko T and Hyodo I: Alcohol is an oxidative stressor for gastric epithelial cells: Detection of superoxide in living cells. J Clin Biochem Nutr 53: 75-80, 2013.

20. Bindokas VP, Jordán J, Lee CC and Miller RJ: Superoxide production in rat hippocampal neurons: Selective imaging with hydroethidine. J Neurosci 16: 1324-36, 1996.

21. Toyokuni S, Tanaka T, Hattori Y, Nishiyama Y, Yoshida A, Uchida K, Hiai A, Ochi A and Osawa T: Quantitative immunohistochemical determination of 8-hydroxy-2'deoxyguanosine by a monoclonal antibody N45.1: Its application to ferric nitrilotriacetate-induced renal carcinogenesis model. Lab Invest 76: 365-374, 1997.

22. Vermes I, Haanen C, Steffens-Nakken $H$ and Reutelingsperger C: A novel assay for apoptosis. Flow cytometric detection of phosphatidylserine expression on early apoptotic cells using fluorescein labelled Annexin V. J Immunol Methods 17: 39-51, 1995.

23. Britigan BE, Cohen MS and Rosen GM: Detection of the production of oxygen-centered free radicals by human neutrophils using spin trapping techniques: A critical perspective. J Leukoc Biol 41: 349-362, 1987.

24. Mahmoud SS and Croteau RB: Strategies for transgenic manipulation of monoterpene biosynthesis in plants. Trends Plant Sci 7: 366-373, 2002.

25. Gupta SC, Prasad S, Sethumadhavan DR, Nair MS, Mo YY and Aggarwal BB: Nimbolide, a limonoid triterpene, inhibits growth of human colorectal cancer xenografts by suppressing the proinflammatory microenvironment. Clin Cancer Res 19: 4465-4476, 2013.

26. Greay SJ, Ireland DJ, Kissick HT, Levy A, Beilharz MW, Riley TV and Carson CF: Induction of necrosis and cell cycle arrest in murine cancer cell lines by Melaleuca alternifolia (tea tree) oil and terpinen-4-ol. Cancer Chemother Pharmacol 65: 877-888, 2010.

27. Brand C, Ferrante A, Prager RH, Riley TV, Carson CF, Finlay-Jones JJ and Hart PH: The water-soluble components of the essential oil of Melaleuca alternifolia (tea tree oil) suppress the production of superoxide by human monocytes, but not neutrophils, activated in vitro. Inflamm Res 50: 213-219, 2001.

28. Kim HJ, Chen F, Wu C, Wang X, Chung HY and Jin Z: Evaluation of antioxidant activity of Australian tea tree (Melaleuca alternifolia) oil and its components. J Agric Food Chem 52: 2849-2854, 2004.

29. Schumacker PT: Reactive oxygen species in cancer cells: live by the sword, die by the sword. Cancer Cell 10: 175-176, 2006.

30. Nogueira V and Hay N: Molecular pathways: Reactive oxygen species homeostasis in cancer cells and implications for cancer therapy. Clin Cancer Res 19: 4309-4314, 2013.

31. Glasauer A, Sena LA, Diebold LP, Mazar AP and Chandel NS: Targeting SOD1 reduces experimental non-small-cell lung cancer. J Clin Invest 124: 117-128, 2014. 
32. Provinciali M, Donnini A, Argentati K, Di Stasio G, Bartozzi B and Bernardini G: Reactive oxygen species modulate $\mathrm{Zn}(2+)$-induced apoptosis in cancer cells. Free Radic Biol Med 32: 431-445, 2002.

33. Kuo PL, Chen CY and Hsu YL: Isoobtusilactone A induces cell cycle arrest and apoptosis through reactive oxygen species/apoptosis signal-regulating kinase 1 signaling pathway in human breast cancer cells. Cancer Res 67: 7406-7420, 2007.

34. Whibley CE, McPhail KL, Keyzers RA, Maritz MF, Leaner VD, Birrer MJ, Davies-Coleman MT and Hendricks DT: Reactive oxygen species mediated apoptosis of esophageal cancer cells induced by marine triprenyl toluquinones and toluhydroquinones. Mol Cancer Ther 6: 2535-2543, 2007.

35. Trachootham D, Zhou Y, Zhang H, Demizu Y, Chen Z, Pelicano H, Chiao PJ, Achanta G, Arlinghaus RB, Liu J and Huang P: Selective killing of oncogenically transformed cells through a ROS-mediated mechanism by beta-phenylethyl isothiocyanate. Cancer Cell 10: 241-252, 2006

36. Ka H, Park HJ, Jung HJ, Choi JW, Cho KS, Ha J and Lee KT: Cinnamaldehyde induces apoptosis by ROS-mediated mitochondrial permeability transition in human promyelocytic leukemia HL-60 cells. Cancer Lett 196: 143-152, 2003.

37. Noh J, Kwon B, Han E, Park M, Yang W, Cho W, Yoo W, Khang G and Lee D: Amplification of oxidative stress by a dual stimuli-responsive hybrid drug enhances cancer cell death. Nat Commun 6: 6907, 2015.

38. Avisetti DR, Babu KS and Kalivendi SV: Activation of p38/JNK pathway is responsible for embelin induced apoptosis in lung cancer cells: Transitional role of reactive oxygen species. PLoS One 9: e87050, 2014.
39. Ichijo H, Nishida E, Irie K, ten Dijke P, Saitoh M, Moriguchi T, Takagi M, Matsumoto K, Miyazono K and Gotoh Y: Induction of apoptosis by ASK1, a mammalian MAPKKK that activates SAPK/JNK and p38 signaling pathways. Science 275: 90-94, 1997.

40. Toyokuni T and Akatsuka S: What has been Learned from the Studies of Oxidative Stress-induced Carcinogenesis: Proposal of the Concept of Oxygenomics. J Clin Biochem Nutr 39: 3-10, 2006.

41. Perry G, Castellani RJ, Hirai K and Smith MA: Reactive oxygen species mediate cellular damage in alzheimer disease. J Alzheimers Dis 1: 45-55, 1998.

42. Kim YW and Byzova TV: Oxidative stress in angiogenesis and vascular disease. Blood 123: 625-631, 2014.

43. Rolo AP, Teodoro JS and Palmeira CM: Role of oxidative stress in the pathogenesis of nonalcoholic steatohepatitis. Free Radic Biol Med 52: 59-69, 2012.

44. Forbes JM, Coughlan MT and Cooper ME: Oxidative stress as a major culprit in kidney disease in diabetes. Diabetes 57: 1446-1454, 2008.

45. Berndtsson M,Hägg M, Panaretakis T, Havelka AM, Shoshan MC and Linder S: Acute apoptosis by cisplatin requires induction of reactive oxygen species but is not associated with damage to nuclear DNA. Int J Cancer 120: 175-180, 2007. 